\title{
Decomposition of the Visible and Dark Matter Mass Profiles in the Einstein Ring 0047-2808
}

\author{
Simon Dye \& Steve Warren \\ Astrophysics Group, Imperial College London, Blackett Labs, Prince \\ Consort Road, London, SW7 $2 B W$, U.K.
}

\begin{abstract}
Using our semi-linear inversion method, we measure the mass profile of the lens galaxy in the Einstein ring system 0047-2808. The lens is modeled as a baryonic component following the observed light embedded in a generalised dark matter NFW halo. The semilinear method makes full use of the information content in the ring image. We determine a B-band mass to light ratio for the baryons of $2.33_{-0.62}^{+0.30} h M_{\odot} / L_{B \odot}(99 \% \mathrm{CL})$, accounting for $54 \%$ of the total projected mass within the Einstein radius of $1.16^{\prime \prime}$. The inner logarithmic slope of the halo is found to be $0.65_{-0.31}^{+0.73}(99 \% \mathrm{CL})$. We find that the halo is fairly well aligned with the light but has only half the ellipticity.
\end{abstract}

\section{Introduction}

Gravitational lensing provides a simple and well understood tool for the determination of galaxy mass profiles. It has the advantage that the deflection angle of a photon passing a massive object is independent of the dynamical state of the deflecting mass. Dynamical methods for measuring galaxy mass profiles rely on a number of assumptions that can be difficult to test, such as dynamical equilibrium, circular orbits of gaseous disks, and, frequently, spherical symmetry of the mass distribution.

In contrast to weak lensing, where a statistical detection of galaxy-galaxy lensing can place limits on mass profiles (eg. Hoekstra, Yee, \& Gladders 2003), strong lensing involves the analysis of individual systems and allows smaller scales to be probed. In strong lens systems, multiple images of a background source are observed. Once the redshift of the source and lens galaxy are known, mass models for the lens can be fit by comparing predicted image positions with those measured. Strong lens systems where the source has extended structure give rise to multiple arc-shaped images. These arcs trace out the Einstein ring which, as Kochanek, Keeton, \& McLeod (2001) show, allows stronger constraints to be placed on the lens mass profile. Unlike strong lens systems where the source is point-like, the analysis of Einstein rings requires modeling of the source surface brightness distribution. In this way, the properties of both the source and the lens are minimised to give the best fit to the observed ring.

In the analysis of Einstein rings, the way in which the source is modelled has a significant effect. Assuming a simple analytical surface brightness profile for the source can bias the lens model solution. Real sources typically have 
complicated structure and an over-simplified source forces the fitted lens model to compensate. A non-parametric form, such as one in which the source surface brightness is pixelised, almost entirely removes this problem but creates a new one: The surface brightness in each source pixel must be adjusted in addition to the lens model parameters to find the minimum thus hugely slowing the procedure.

Wallington, Kochanek, \& Narayan (1996) describe a method based on maximum entropy that uses a pixelised source surface brightness distribution. This has been applied by Wayth et al. (2003) to the Einstein ring 0047-2808. At the heart of the method are two nested minimisation cycles. For a given lens model stipulated by the outer cycle, the surface brightnesses in the source pixels are adjusted in the inner cycle until an image is obtained which gives the best fit to the observed ring image. The inner cycle typically needs hundreds of iterations before satisfactory convergence is obtained.

In a previous paper (Warren \& Dye 2003), we developed a new technique called the semi-linear method. The semi-linear method uses a pixelised source plane but replaces the inner cycle of the maximum entropy method with a singlestep linear inversion. This has the advantage that it is very fast and, more importantly, that the correct source minimum is guaranteed for a given lens model. The possibility of minimising to an incorrect source, thereby biasing the minimised lens model, is thus completely removed.

In the work presented here, we apply the semi-linear method to WFPC2 observations of the Einstein ring 0047-2808. We model the lens as a baryonic component embedded in a dark halo and show how the contribution from each component can be determined to allow measurement of the baryonic M/L.

\section{The semi-linear reconstruction method}

We refer the reader to Warren \& Dye (2003) for a complete description of the semi-linear method. Here, we give an outline.

The inversion requires that both the source plane and the image plane are pixelised. This pixelisation can be of a completely general fashion in terms of the distribution of pixel sizes and locations. Labeling the surface brightness in reconstructed source pixel $i$ as $s_{i}$, the flux in observed image pixel $j$ as $d_{j}$ and the $1 \sigma$ error on this flux as $\sigma_{j}$, then for a given lens model, the vector of source surface brightnesses which best fits the observed ring is given by

$$
\mathbf{s}=(\mathbf{F}+\lambda \mathbf{R})^{-1} \mathbf{t}
$$

where

$$
\mathrm{F}_{k l}=\sum_{j=1}^{N} f_{k j} f_{l j} / \sigma_{j}^{2}, \quad \mathrm{R}_{k l}=2 \sum_{i=1}^{M} r_{i k} r_{i l} \quad \text { and } \quad t_{i}=\sum_{j=1}^{N} f_{i j} d_{j} / \sigma_{j}^{2} .
$$

The quantity $f_{i j}$ is the contribution to the flux in image pixel $j$ from source pixel $i$ according to the given lens model and allowing for smearing by the observed image PSF. The solution is regularised by the matrix $\mathbf{R}$, the form of which is selected by the user. This is chosen to reduce noise in the reconstructed source by penalising spikes with the regularisation weight $\lambda$ (see Warren \& Dye 2003). 
Equation (1) gives the best fit source for a fixed lens model. This is the linear part of the semi-linear method. The non-linear part comes from the need to adjust the lens parameters in an outer iterative cycle until the best global fit is obtained. At each step in the outer cycle, a new set of quantities $f_{i j}$ must be calculated.

\section{Data}

Full details of the HST observations and data reduction steps are given in Wayth et al. (2003).

The data consist of drizzled images of the Einstein ring 0047-2808 observed with the WFPC2 instrument. The source in this system is a star-forming galaxy at redshift $z=3.595$. The galaxy has strong Ly $\alpha$ emission (Warren et al. 1996), hence observations were carried out in the F555W filter to maximise the ring:lens flux ratio. The fully reduced, drizzled image $(2 \times 2$ dither positions per full WFPC2 pixel) is shown in the left of Figure 1.
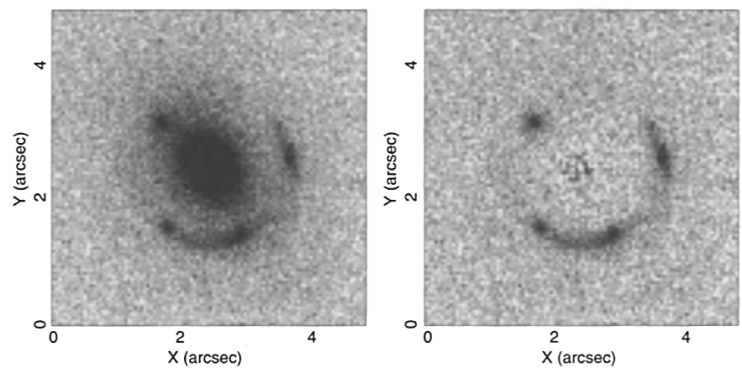

Figure 1. Drizzled WFPC2 observation of 0047-2808 in F555W filter. Left: Original image Right: Ring with lensing galaxy subtracted (small central residuals not significant)

Since the semi-linear method requires an image of the ring only, the lens galaxy had to be first removed. The best fitting surface brightness distribution for the lens galaxy was found to be a Sersic profile (Sersic 1968) with a central point source (Wayth et al. 2003). The plot on the right of Figure 1 shows the resulting Einstein ring after the lensing galaxy has been subtracted. The residue at the centre of the subtracted image is insignificant Poisson noise.

\section{Modeling procedure}

In our dual component lens model, we wish to constrain the inner slope of the density profile of the dark halo and the baryonic mass-to-light ratio.

The baryonic component of the lens model is assumed to follow the light of the lens galaxy, fixed by the fitted Sersic + point source profile. The lens deflection angle due to the baryons is therefore calculated from this light profile scaled by the B-band baryonic mass to light ratio $\Psi$ (in units of $h M_{\odot} / L_{B \odot}$ ). 
The surface density of the Sersic profile in units of $M_{\odot} / \square^{\prime \prime}$, is

$$
\Sigma_{b}(\eta)=\Psi L_{1 / 2} \exp \left\{-5.90\left[\left(\eta / 1.31^{\prime \prime}\right)^{0.32}-1\right]\right\}
$$

and the mass of the central point source,

$$
M_{p}=3.09 \times 10^{9} h^{-2} L_{\odot} \Psi,
$$

expressed here in units of $M_{\odot} . \Psi$ is the only free parameter describing the baryonic contribution to the total lens mass. The half light luminosity of the Sersic profile is $L_{1 / 2}=2.33 \times 10^{9} h^{-2} L_{B \odot} / \square^{\prime \prime}$ and the ellipse co-ordinate $\eta^{2}=$ $x^{2}+y^{2} / q^{2}$ has a measured axis ratio $q=b / a=0.69$. The orientation of the major axis to the vertical in a counter-clockwise direction is $35^{\circ}$.

The dark matter halo is modelled with a generalised NFW model (Navarro, Frenk, \& White 1996) with the central density profile slope $\gamma$ left as a free parameter. This has a density profile given by

$$
\rho(r)=\frac{\rho_{s}}{\left(r / r_{s}\right)^{\gamma}\left(1+r / r_{s}\right)^{3-\gamma}}
$$

Here, $\rho_{s}$ is the halo normalisation and $r_{s}$ is a scale radius. The volume mass density $\rho(r)$ must be converted to a projected surface mass density profile by integrating along the line of sight. We generalise this surface mass density to an elliptical distribution using the ellipse co-ordinate $\eta$. Following the prescription given by Keeton (2001), the elliptical surface density is used to calculate the deflection angle.

The parameters describing the lens model are then the baryonic M/L $\Psi$, the position of the halo centre with respect to the light centre $\left(x_{h}, y_{h}\right)$, the halo normalisation $\rho_{s}$, the central slope $\gamma$, the halo ellipticity $e_{h}=1-b / a$ and the orientation of the semi-major axis to the vertical in a counter-clockwise direction, $\theta_{h}$. We hold the scale radius fixed at $r_{s}=40 h^{-1} \mathrm{kpc}\left(\cong 9^{\prime \prime} @ z=0.485, \Omega=\right.$ $0.3, \Lambda=0.7)$ to match that expected from simulations by Bullock et al. (2001) for a galaxy of equal mass and redshift of the lens galaxy in 0047-2808. The fitted lens model is relatively insensitive to the choice of $r_{s}$.

Joint confidence contours are obtained on the $\Psi, \gamma$ plane by minimising over the remaining 5 parameters at regular grid points in the plane. We use a modified version of Powell's method (Press et al. 2001) for the minimisation. We mask the ring with an annulus to remove as many insignificant sky pixels as possible (see Figure 2).

\section{Results}

Figure 2 shows the results of the minimisation. The confidence regions on $\Psi$ and $\gamma$ are shown in the plot on the left. In order not to bias the lens solution, this minimisation was not regularised (ie. $\lambda$ was set to 0 ). We used a source plane size of $0.5^{\prime \prime} \times 0.5^{\prime \prime}$ with an adaptive pixelisation scheme dependent on the lens magnification (see Dye \& Warren, 2003). To $99 \%$ confidence, we measure an inner slope of $\gamma=0.65_{-0.31}^{+0.73}$ and a baryonic $\mathrm{M} / \mathrm{L}$ of $\Psi=2.33_{-0.62}^{+0.30} h M_{\odot} / L_{B \odot}$. The dashed lines show the same confidence levels obtained by Koopmans \& Treu 
(2003) in analysing the same HST observations as those here. These confidence levels were obtained using only the eight constraints provided by the four multiple image positions. ${ }^{1}$ Clearly, by using the full information content of the ring image, the semi-linear method gives substantially better constraints.

The other minimised lens model parameters are $\left(x_{h}, y_{h}\right)=\left(0.00^{\prime \prime}, 0.02^{\prime \prime}\right)$, $e_{h}=0.136 \pm 0.02$ and $\theta_{h}=40.7 \pm 2.4^{\circ}$, which give $\chi_{\nu}^{2}=0.977 \pm 0.039$. The centre of the halo is well aligned with the centre of the light whereas the orientation is marginally inconsistent at the $\sim 2 \sigma$ level. The halo is much rounder than the light distribution with an ellipticity less than half the value of $e=0.31$ for the Sersic profile. Finally, the halo normalisation is such that its contribution to the total projected mass within the Einstein radius of $1.16^{\prime \prime}$ (Wayth et al. 2003) is $46 \%$.
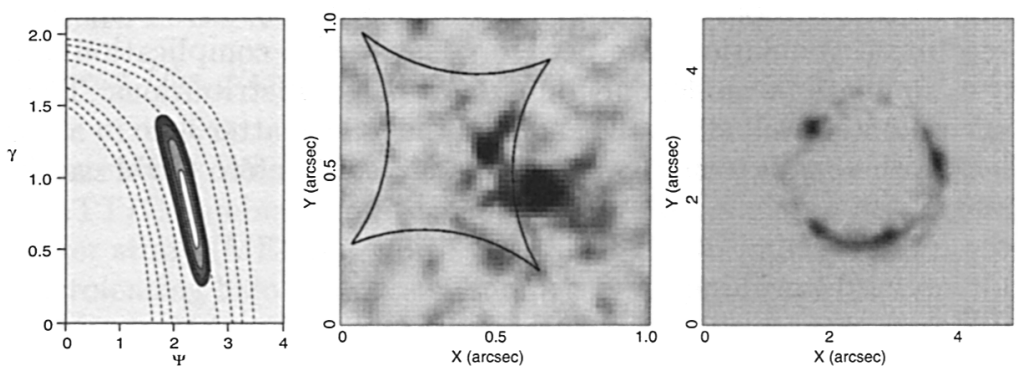

Figure 2. Left: $68 \%, 95 \%, 99 \% \& 99.9 \%$ confidence regions on inner slope $\gamma$ and baryonic $\mathrm{M} / \mathrm{L}, \Psi$. Dashed lines are same confidence regions from lens analysis by Koopmans \& Treu (2003) (excluding dynamical information) Middle: Regularised reconstructed source pair showing lens caustic (note different scale). Right: Image of regularised source in masked annular region.

The middle plot of Figure 2 shows the reconstructed source. To best resolve the source morphology we performed a regularised inversion, fixing the parameters of the lens model to those obtained above for the unregularised solution. $\lambda$ was set to the value $\operatorname{Tr}(\mathbf{F}) / \operatorname{Tr}(\mathbf{R})$ so that both matrices in equation (1) were weighted equally. Although this gives a ring image (on the right in Figure 2) with a value of $\chi^{2}$ that is worse than in the unregularised case, the reconstructed source allows two distinct objects to be identified. This agrees with the findings of Wayth et al. (2003) and illustrates the importance of using a non-parametric source: If a single, analytic source had been used instead, the minimised lens model would have been biased in this case.

\section{Summary \& Discussion}

We have applied our semi-linear method to HST observations of the Einstein ring system 0047-2808 to determine the lens galaxy mass profile. Modeling the lens as a baryonic Sersic profile + point source nested in a dark matter generalised NFW halo, we find a $\mathrm{M} / \mathrm{L}$ of $2.33_{-0.62}^{+0.30} h M_{\odot} / L_{B \odot}$ and an inner slope

\footnotetext{
${ }^{1}$ They derived somewhat improved constraints by using also the dynamical information provided by the measured stellar velocity dispersion.
} 
$\gamma=0.65_{-0.31}^{+0.73}(99 \%$ CL). The reconstructed source surface brightness distribution shows two distinct source objects, highlighting the need for non-parametric sources to obtain unbiased lens mass profiles.

One of the main predictions of the Cold Dark Matter (CDM) model is that halo mass profiles are cuspy. Recent CDM simulations show that the inner slope should lie somewhere in the range $1 \lesssim \gamma \lesssim 1.5$ (Navarro, Frenk, \& White 1996; Moore et al. 1999). Our measurement of $\gamma$ for 0047-2808 is not inconsistent with these. Nevertheless there are two important factors which must be considered. The first is that the simulations are only just starting to reliably probe scales of $\sim$ few kpc. The most recent simulations seem to indicate that $\gamma$ continues to decrease at smaller radii with no sign of reaching a convergent value (Power et al. 2003; see also Navarro et al. in these proceedings). In order to feel completely comfortable when comparing these predictions with observational constraints, higher resolution simulations are needed. The second complication is that the majority of simulations model only pure dark matter distributions. The effect of a baryonic potential well alters the shape of the dark matter halo in a non-trivial way. Clearly, these shortcomings must be addressed before CDM can be tested with confidence.

\section{References}

Bullock, J.S., Kollat, T.S., Sigad, Y., Somerville, R.S., Kravtsov, A.V., Klypin, A.A., Primack, J.R., Deckel, A., 2001, MNRAS, 321, 598

Dye S. \& Warren S.J., 2003, ApJ, submitted

Hoekstra, H., Yee, H.K.C., Gladders, M.D., 2003, astro-ph/0306515

Keeton, C.R., 2001, astro-ph/0102341

Kochanek, C.S., Keeton, C.R., \& McLeod, B.A., 2001, ApJ, 547, 50

Koopmans, L.V.E. \& Treu, T., 2003, ApJ, 583, 606

Moore, B., Quinn, T., Governato, F., Stadel, J., Lake, G, 1999, MNRAS, 310, 1147

Navarro, J.F., Frenk, C.S., \& White S.D.M., 1996, ApJ, 462, 563

Power, C., Navarro, J.F., Jenkins, A., Frenk, C.S., White, S.D.M., Springel, V., Stadel, J., Quinn, T., 2003, MNRAS, 338, 14

Press, W.H., Teukolsky, S.A., Vetterling, W.T., Flannery, B.P., 2001, 'Numerical Recipes in Fortran 77, 2nd Edition', Cambridge University Press

Sersic, J.L., 1968, Cordoba, Argentina: Observatorio Astronomico (1968)

Wallington, S., Kochanek, C.S., \& Narayan, R., 1996, ApJ, 465, 64

Warren, S.J., Hewett, P.C., Lewis, G.F., Møller, P., Iovino, A., Shaver, P.A., 1996, MNRAS, 278, 139

Warren, S.J. \& Dye, S., 2003, ApJ, 590, 673

Wayth, R.B., Warren, S.J., Lewis, G.F., Hewett, P.C., 2003, MNRAS, in prep 